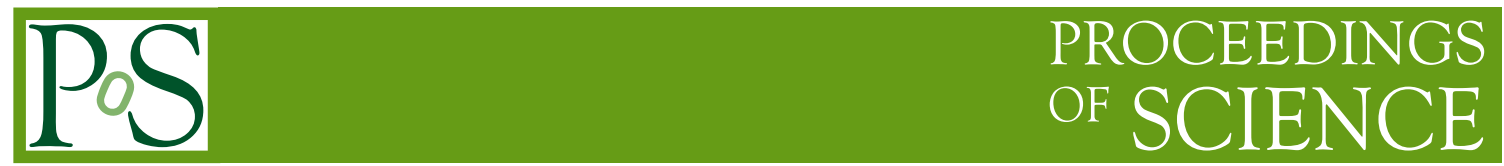

\title{
Heavy-Flavor Transport and Hadronization in the QGP
}

\author{
Ralf $\operatorname{Rapp}^{a, *}$ \\ ${ }^{a}$ Texas A\&M University \\ Cyclotron Institute and Department of Physics \& Astronomy, MS 3366, College Station, TX 77843-3366, \\ USA \\ E-mail: rapp@comp.tamu.edu
}

\begin{abstract}
A survey is given on recent developments in using heavy-flavor observables to probe the quarkgluon plasma and its hadronization in ultrarelativistic heavy-ion collisions. Emphasis is placed on trying to gain insights into the medium modifications of the fundamental QCD force. Where appropriate, the role of open and hidden bottom particles is highlighted.
\end{abstract}

BEAUTY2020

21-24 September 2020

Kashiwa, Japan (online)

\footnotetext{
${ }^{*}$ Speaker
} 


\section{Introduction}

The fundamental QCD force between a heavy quark $(Q=b, c)$ and its antiquark is well established in terms of the originally proposed Cornell potential which is characterized by a shortrange color-Coulomb and a long-range confining interaction,

$$
V(r)=-\frac{4}{3} \frac{\alpha_{s}}{r}+\sigma r .
$$

It has been quantitatively confirmed in lattice-QCD (1QCD) calculations and provides a solid starting point to understand the spectroscopy of charmonia and bottomonia, with systematic corrections due to spin-orbit and spin-spin interactions calculable through higher orders in an expansion of the inverse heavy-quark (HQ) mass, $1 / m_{Q}$ [1]. The linear part of the potential, associated with a nonperturbative string tension of about $\sigma \simeq 1 \mathrm{GeV} / \mathrm{fm}$, remains one of the most direct phenomenlogical manifestations of quark confinement in QCD. Except for the ground-state bottomonia $\left(\eta_{b}\right.$ and $\Upsilon(1 S)$ ), quarkonia derive most of their binding from the confining force. This renders them excellent probes of deconfinement in hot and dense QCD matter as produced in ultrarelativistic heavy-ion collisions (URHICs), see, e.g., Refs. [2-5]. In addition, open heavy-flavor (HF) particles turn out to be an ideal tool to study the transport properties of the QCD medium [6, 7]: their low-momentum Brownian motion in the medium modifies their transverse-momentum $\left(p_{T}\right)$ spectra in URHICs relative to those in elementary proton-proton $(p p)$ collisions, which can be utilized to quantify the HF diffusion coefficients. Furthermore, the conversion of heavy quarks into different HF hadrons near the phase transition can inform us about mechanisms of hadronization. In the following, we first discuss theoretical foundations and recent insights into the properties of HF particles in equilibrium matter (Sec. 2), then highlight recent developments in URHIC phenomenology of open HF (Sec. 3) as well as quarkonia (Sec. 4), and conclude in Sec. 5.

\section{Heavy Flavor in Medium}

In this section we discuss the properties of HF particles in QCD matter at finite temperature, thereby emphasizing their special role in applications to URHICs, first for quarkonia (Sec. 2.1) and then for open heavy flavor (Sec. 2.2).

\subsection{Quarkonia}

The key quantity to analyze the in-medium properties of quarkonia are their spectral functions. The peak positions encode the information on their in-medium binding energy, $E_{B}(p, T)$ (depending on both temperature, $T$, and 3-momentum $p$ ), in connection with medium modifications of the HQ mass, $m_{Q}(T)$. The widths of the peaks provide information on the inelastic reaction rate, $\Gamma_{\alpha}\left(p, T ; E_{B}\right)$, a key transport parameter for URHIC phenomenolgy which itself depends on the binding energy ( $\alpha=\Psi, Y$ generically denotes a charmonium or bottomonium state). Quarkonium spectral functions play an important role as an interface between first-principle 1QCD results and URHICs. As IQCD computations are carried out in euclidean space-time, this interface is usually realized through model (or effective-theory) calculations. The large HQ mass implies the applicability of potential approximations, as the energy transfer is parametrically suppressed 

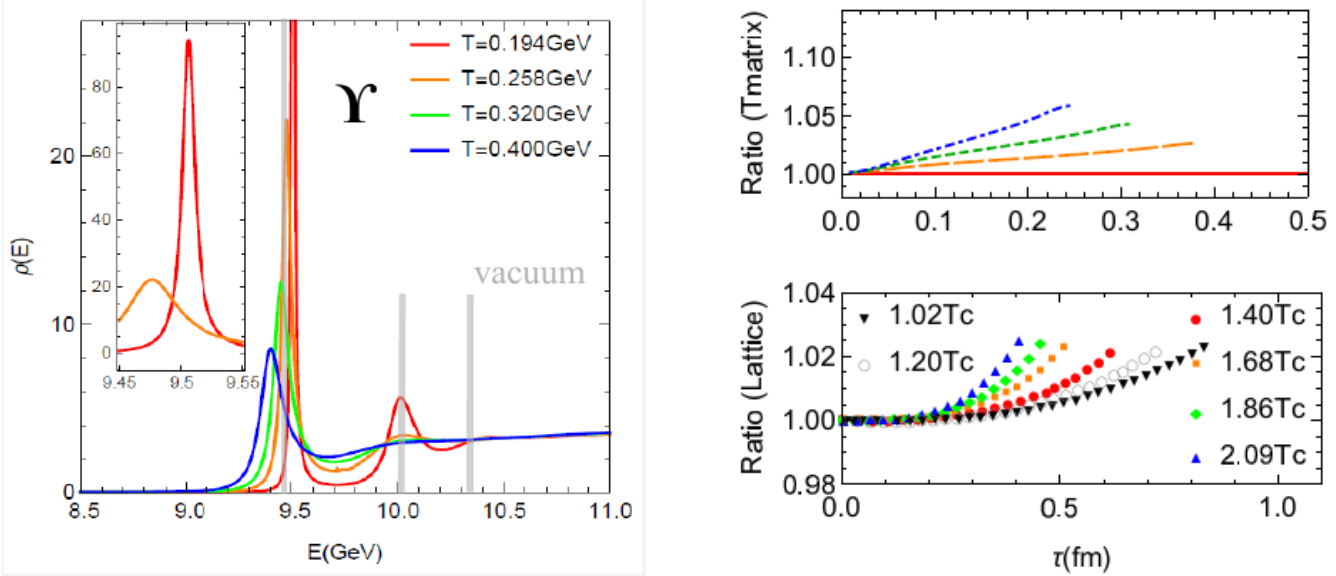

Figure 1: Theoretical calculations of the in-medium bottomonium spectral function in the vector channel $\left(J^{P}=1^{-}\right)$within the $T$-matrix approach [8] (left) and the ratio of the pertinent euclidean correlators relative to the one at the lowest temperature (upper right), in comparison to lQCD data [9] (lower right).

compared to the momentum transfer, $q_{0} \sim q^{2} / 2 m_{Q} \ll q$. A recent example is illustrated in Fig. 1 left showing results for in-medium bottomonium spectral functions from the thermodynamic $T$ matrix formalism [8]. The latter utilizes an in-medium HQ potential that also describes the HQ free energy as computed in IQCD (the potential, is, however, substantially larger than the free energy due to the presence of selfconsistently calculated imaginary parts, i.e., scattering rates). One finds a sequential dissocation of the $\Upsilon(3 S)$ near the crossover transition temperature, $T_{\mathrm{c}}$, the $\Upsilon(2 S)$ at $T \simeq 200 \mathrm{MeV}$, and the $\Upsilon(1 S)$, surviving to much higher $T$, beyond $400 \mathrm{MeV}$, albeit with a gradually increasing width. The right panel of Fig. 1 shows that the ratios of euclidean correlation functions computed from the $T$-matrix (upper panel) compare fairly well to lQCD results [9] (lower panel).

A basic building block to describe quarkonia in the quark-gluon plasma (QGP), especially when they are loosely bound, is the interaction of the individual heavy quarks within the bound state with the thermal-medium partons (with small corrrections from recoil effects associated with $\left.E_{B}\right)$, cf. Fig. 2. This demonstrates the close connection between in-medium quarkonia and the propagation of single heavy quarks in the QGP, which becomes explicit in a quarkonium scattering equation,

$$
T_{Q \bar{Q}}=V_{Q \bar{Q}}+\int V_{Q \bar{Q}} D_{Q} D_{\bar{Q}} T_{Q \bar{Q}}
$$

( $D_{Q}$ : HQ propagator). A good understanding of HQ properties in the QGP is therefore mandatory to reliably describe quarkonia, and is arguably a simpler problem which we turn to next.

\subsection{Open Heavy Flavor}

The large HQ mass, relative to the typical temperatures reached in URHCs, implies that lowmomentun $c$ and $b$ quarks diffuse via Brownian motion through elastic collisons with thermal partons (note that the potential approximation remains valid for heavy-light scattering, similar to a tennis ball bouncing off a wall where essentially no energy transfer occurs). Furthermore, the HQ thermalization time, $\tau_{Q}$, is delayed relative to light partons, parametrically by $m_{Q} / T$, which renders it comparable to (for charm) or even larger than (for bottom) the QGP lifetime. Thus HQ momentum 

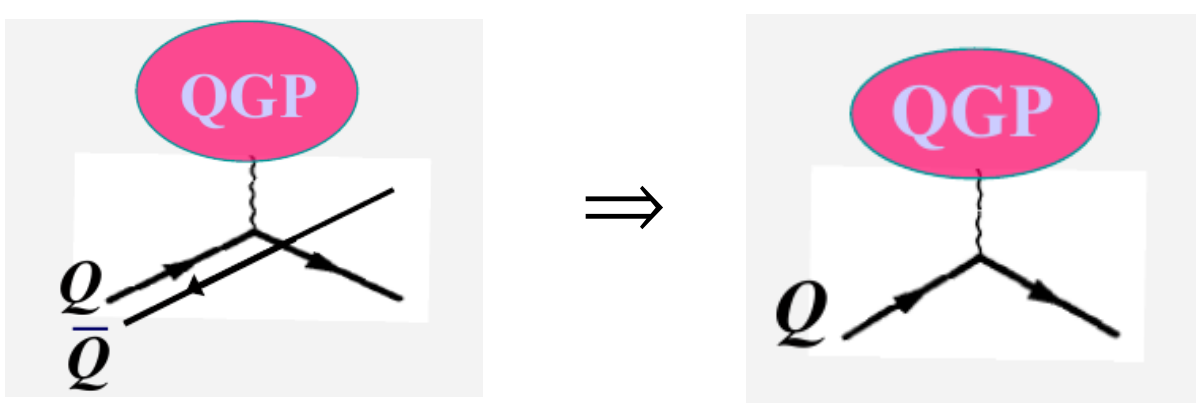

Figure 2: Illustration of the relation between inelastic quarkonium interactions (left) and elastic scattering of a single heavy quark (right) in the QGP.

spectra in URHICs carry a memory of their interaction history which provides an excellent gauge of their interaction strength with the medium and thus direct access to the HF diffusion coefficient. The latter is defined as the zero-momentum limit of the thermal relaxation time, $\mathcal{D}_{s}=\tau_{Q} T / m_{Q}$, where the $T / m_{Q}$ takes out, in first approximation, the HQ kinematics so that $\mathcal{D}_{s}$ is approximately independent of $m_{Q}$ (see Ref. [10] for a discussion of this point). Accounting for another factor of $\sim 3$ between scattering and relaxation time, the HQ diffusion coefficient directly relates to the collisional width of heavy quarks. When applying this to the light-parton sector, one obtains an estimate of the thermal-parton widths and thus information on QGP structure. A powerful feature of the potential approach is its ability to include nonperturbative effects, both in terms of the input potential and the resummation of the ladder series. Remnants of the confining force above $T_{\mathrm{c}}$ are, in fact, a leading candidate for generating the interaction strength needed for a strongly-coupled QGP ("sQGP" or "near-perfect liquid"), while resummations are inevitable for bound-state formation, i.e., hadronization. The formation of different open $\mathrm{HF}$ hadrons $\left(D, D_{s}, \Lambda_{c}, B, B_{s}, \Lambda_{b}\right.$, etc.) from a universal underlying HQ distribution function transported through the QGP, is therefore a good probe of hadronization mechanisms.

\section{Open HF Transport in URHICs}

The transport of heavy quarks (hadrons) through the QGP (hadronic matter) of the fireball in URHICs is commonly described by simulating the HQ phase space distribution function, $f_{Q}$, by either a relativistic Boltzmann (BM) or Fokker-Planck (FP) equation schematically given by

$$
\frac{\partial}{\partial t} f_{Q}(t, p)=\gamma_{Q} \frac{\partial}{\partial p_{i}}\left[p_{i} f_{Q}(t, p)\right]+D_{p} \frac{\partial^{2}}{\partial^{2} p} f_{Q}(t, p) .
$$

The thermalization rate $\gamma_{Q}=1 / \tau_{Q}$ and momentum diffusion coefficient $D_{p}$, related via $D_{p}=$ $\gamma_{Q} m_{Q} T$, are moments of the momentum transfer $q$ convoluted over the (squared) heavy-light scattering amplitude and thermal disitribution functions of the light medium constituents. The FP equation can be derived from the BM equation in an expansion of $q / p$ (which is of odrer $\sqrt{T / m_{Q}}$ ). However, the transport coefficients in the FP equation can be calculated more generally including the quantum effects (spectral functions) of the medium partons [11], which is not straightforward in the semi-classical BM equation. A scenario of the sQGP where the light-parton spectral fucntions are "melted" while the heavy quarks are still reasonable quasi-particles is quite conceivable for 

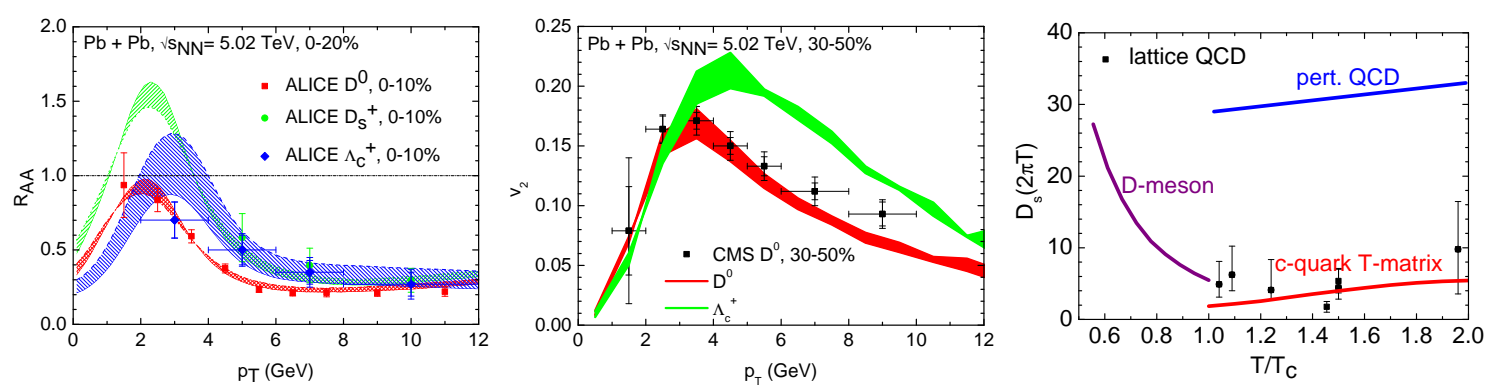

Figure 3: LHC data for the $R_{\mathrm{AA}}[12,13]$ (left) and $v_{2}$ [14] (middle) of charm hadrons in $\mathrm{Pb}-\mathrm{Pb}(5.02 \mathrm{TeV}$ ) collisions, compared to transport calculations [15] with a charm diffusion coefficient shown in the right panel.

temperatures up to $2 T_{\mathrm{c}}$ or so [8]. In the transition from QGP to hadronic matter, heavy quarks need to hadronize. There is consensus that recombination with thermal partons plays a critical role in this process, especially at low momenta, possibly up to $p \simeq 5-10 \mathrm{GeV}$ [6], beyond which fragmentation takes over. Heavy-hadron diffusion in the hadronic phase probably has a rather small, but not negligible, effect on the final observables.

Typical observables for HF hadrons in URHICs are the nuclear modification factor, $R_{\mathrm{AA}}$, and elliptic flow coefficient, $v_{2}$. The former is the ratio of the yield in a heavy-ion collision normalized to the expectation from a mere superposition of primoridal nucleon-nucleon collisions, so that deviations from one quantify medium effects (or initial nuclear effects, such as shadowing). The $v_{2}$ coefficient is the second harmonic of the azimuthal angular modulation of the $p_{T}$ spectrum, which is most relevant for non-central collisions. For light-flavor (bulk) particles, it indicates how effective the medium can convert the initial spatial anisotropy of an almond-shaped fireball into momentum anisotropy via the different pressure gradients along the long and short axis. For HF particles, the low-momentum $v_{2}$ quantifies how much they are dragged along with the hydrodynamic expansion, which is sensitive to their coupling strength to the QCD medium. An example of these observables is shown in Fig. 3, where LHC data [12-14] are compared to model calculations using FP/Langevin simulations based on heavy-light $T$-matrix amplitudes, within a hydrodynamic medium, while hadonization is evaluated within the resonance recombination model [15]. A reasonable description of the $R_{\mathrm{AA}}$ of $D, D_{s}$ (enhanced through the large strange-quark abundance in the QGP) and $\Lambda_{c}$ (enhanced by 3-body recombination) as well as the $D$-meson $v_{2}$ is found. The underlying HQ diffusion coefficient, shown in the right panel of Fig. 3, is similar to other calculations that reproduce the data [6]. Converting that into a scattering rate as discussed in Sec. 2.2, the parametric estimate $\Gamma_{c} \sim 3 m_{c} / T \gamma_{c} \sim 3 / \mathcal{D}_{s}$ yields about $1 \mathrm{GeV}$ at temperatures close to $T_{\mathrm{c}}$, not far from the calculated values of $\sim 0.6 \mathrm{GeV}$ from a selfconsistent $T$-matrix approach [8]. When transferred to the light sector, it implies that the quasi-particle peaks in the low-momentum thermal-parton spectral functions in the sQGP are close to molten. It could also lead to significant quantum corrections for $c$-quark transport, while the heavier $b$ quarks remain better quasi-particles. Future precision data for open-bottom observables are much anticipated, as the theoretical modeling becomes more robust and the degree of thermalization less which increases the sensitivity to the transport coefficients. 

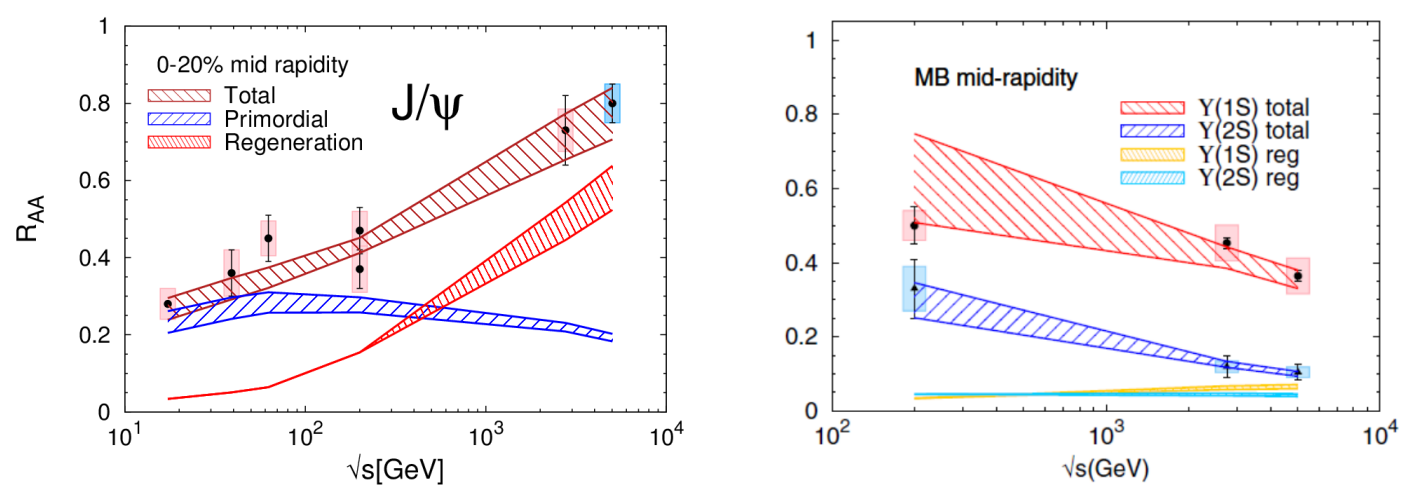

Figure 4: Excitation function of $J / \psi$ (left) and $\Upsilon(1 S), \Upsilon(2 S)$ (right) nuclear modification factors in URHICs.

\section{Quarkonium Transport in URIHCs}

A compact and transparent formulation of a transport description of quarkonia in URHICs is a kinetic rate equation for the number of a quarkonium state, $\alpha$,

$$
\frac{d N_{\alpha}}{d \tau}=-\Gamma_{\alpha}\left[N_{\alpha}-N_{\alpha}^{\mathrm{eq}}\right]
$$

which contains both dissociation and gain terms. The time evolution is governed by two transport parameters, the inelastic reaction rate, $\Gamma_{\alpha}$, and the equilibrium limit, $N_{\alpha}^{\mathrm{eq}}$, which depends on temperature, quarkonium mass and a HQ fugacity factor which accounts for the total abundance of HQ pairs in the fireball (determined by hard production in primordial nucleon-nucelon collisions). The solution of the rate equation requires appropriate initial conditions (depending on the heavy-ion collision energy, $\sqrt{s}$, and centrality) and a model for the space-time evolution of the fireball, usually including QGP, hadronization and hadronic phases.

A synopsis of current quarkonium phenomenology in URHICs is given in Fig. 4 in terms of excitation functions of the $R_{\mathrm{AA}}(\sqrt{s})$ (cf. [23] and references therein). For the $J / \psi$, the $R_{\mathrm{AA}}$ (left panel) shows a marked increase when going from SPS via RHIC to LHC energies: the stronger suppression in the hotter medium is counteracted by a regeneration from recombining $c$ and $\bar{c}$ quarks in the later QGP stages of the fireball evolution (down to $T_{\mathrm{c}}$ ). On the other hand, for $Y$ production, the prevalent effect is a suppression in the hot medium that becomes stronger at larger collision energies, as the regeneration contribution is much smaller. Yet, for $\Upsilon(2 S)$ states, which are almost entirely dissociated at top LHC energy, the relative contribution from regeneration is predicted to be substantial, which may be tested by the shape of their $p_{T}$ spectra.

The better sensitivity of $Y$ states to suppression mechanisms (relative to $\Psi$ states), which largely avoids uncertainties from the regeneration contribution (e.g., the precise determination of the equilibrium limit), opens a more direct window on the in-medium HQ potential, as the reaction rate is sensitive to the binding energy. However, the rate also depends on the heavy-light coupling strength, which is highly nonperturbative as discussed in the previous sections. In Ref. [16], a statistical analysis of available $Y$ data in URHICs has been conducted within a kinetic transport approach using as input a trial potential for the in-medium $Y$ binding energies, in connection with a large $K$ factor for perturbative heavy-light scattering amplitudes. The data fit deduces a rather 

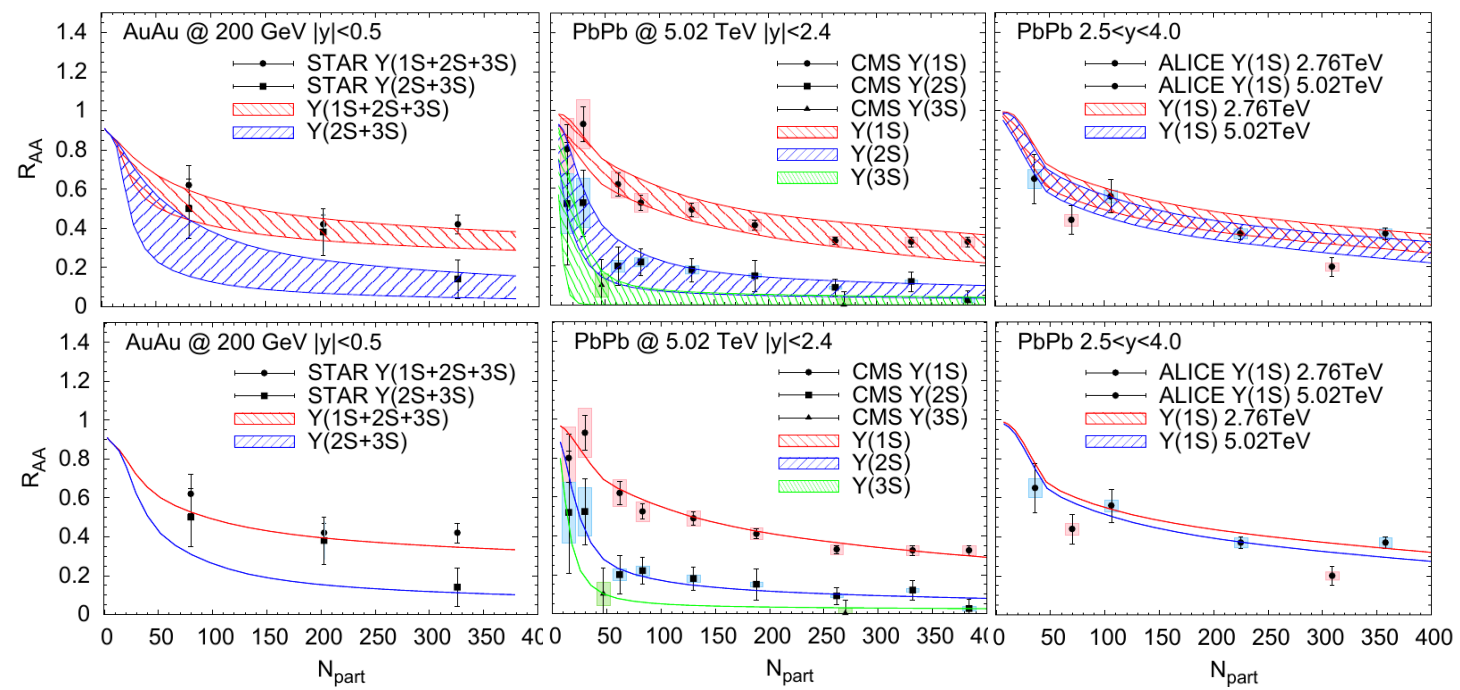

Figure 5: Statistical analysis [16] of bottomonium production in URHICs at RHIC and the LHC using a transport model to extract the in-medium HQ potential. The upper panel shows bands encompassinga a $95 \%$ confidence level, and the lower panel the best fit results. The experimental data are from the STAR [17, 18], ALICE $[19,20]$ and CMS [21, 22] collaborations.

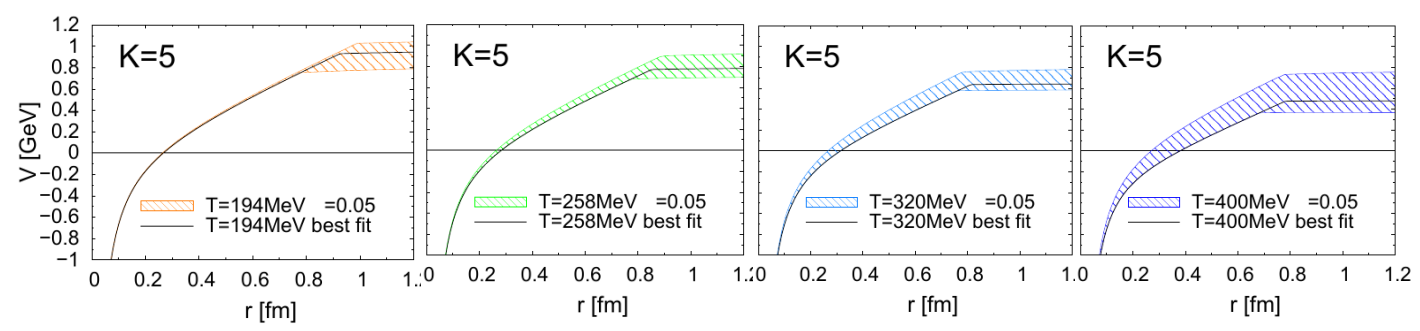

Figure 6: $95 \%$ confidence regions and best fits of the in-medium HQ potential as extracted from a statistical analysis of bottomonium production in URHICs at RHIC and the LHC (cf. Fig. 5).

strong in-medium HQ potential, with substantial remnants of the confining force up to $T \simeq 2.5 T_{\mathrm{c}}$. This potential is remarkably consistent with selfconsistent $T$-matrix calculations [8].

\section{Concluding Remarks}

Heavy-flavor observables in URHICs and their theoretical analyses have reached a maturity that allows to extract quantitative information about transport properties of QCD matter and their underlying microscopic interactions. Utilizing semi-classical transport approaches to describe the Brownian motion of open HF particles in the expanding fireball has revealed that the experimental data for low- and intermediate-momentum charm hadrons require a HQ coupling strength to the QGP that goes far beyond perturbative (color-Coulomb) interactions. This can be naturally explained, in accord with lattice-QCD data, by remnants of the long-range confining force surviving for temperatures of up to at least $2 T_{\mathrm{c}}$. In addition, the chemistry of the observed charm hadrons suggests HQ recombination with thermal partons as an essential hadronization mechanism. Precision measurements in the open-bottom sector will provide crucial tests of this picture. At the same 
time, close connections between the open and hidden HF sector are increasingly being elaborated and exploited. For quarkonia, the large reaction rates in the system may require to go beyond semiclassical transport descriptions; pertinent developments of quantum transport are being developed that will allow to scrutinze conceptual aspects in the interpretation high-quality quarkonium data.

Acknowledgments. This work has been supported the U.S. NSF under grant no. PHY-1913286. I gratefully acknowledge inspiring discussions with Min He.

\section{References}

[1] G. S. Bali, Phys. Rept. 343 (2001) 1.

[2] R. Rapp, D. Blaschke and P. Crochet, Prog. Part. Nucl. Phys. 65 (2010) 209.

[3] P. Braun-Munzinger and J. Stachel, Landolt-Börnstein 23 (2010) 424.

[4] L. Kluberg and H. Satz, Landolt-Börnstein 23 (2010) 372.

[5] A. Mocsy, P. Petreczky and M. Strickland, Int. J. Mod. Phys. A 28 (2013) 1340012.

[6] X. Dong, Y. J. Lee and R. Rapp, Ann. Rev. Nucl. Part. Sci. 69 (2019) 417.

[7] J. Zhao, K. Zhou, S. Chen and P. Zhuang, Prog. Part. Nucl. Phys. 114 (2020) 103801.

[8] S. Y. F. Liu and R. Rapp, Phys. Rev. C 97 (2018) 034918.

[9] G. Aarts et al., JHEP 11 (2011) 103.

[10] S. Y. F. Liu and R. Rapp, Eur. Phys. J. A 56 (2020) 44.

[11] S. Y. F. Liu, M. He and R. Rapp, Phys. Rev. C 99 (2019) 055201.

[12] S. Acharya et al. [ALICE Collaboration], JHEP 1810 (2018) 174.

[13] S. Acharya et al. [ALICE Collaboration], Phys. Lett. B 793 (2019) 212.

[14] A. M. Sirunyan et al. [CMS Collaboration], Phys. Rev. Lett. 120 (2018) 202301.

[15] M. He and R. Rapp, Phys. Rev. Lett. 124 (2020) 042301.

[16] X. Du, S. Y. F. Liu and R. Rapp, Phys. Lett. B 796 (2019) 20.

[17] L. Adamczyk et al. [STAR Collaboration], Phys. Rev. C 94 (2016) 064904.

[18] Z. Ye [STAR Collaboration], Nucl. Phys. A 967 (2017) 600.

[19] B. B. Abelev et al. [ALICE Collaboration], Phys. Lett. B 738 (2014) 361.

[20] S. Acharya et al. [ALICE Collaboration], Phys. Lett. B 790 (2019) 89.

[21] V. Khachatryan et al. [CMS Collaboration], Phys. Lett. B 770 (2017) 357.

[22] A. M. Sirunyan et al. [CMS Collaboration], Phys. Lett. B 790 (2019) 270.

[23] R. Rapp and X. Du, Nucl. Phys. A 967 (2017) 216. 